\title{
Topiramato: efectos secundarios a nivel ocular: Revisión a propósito de un caso
}

\author{
Medin Catoira J. ${ }^{1}$, Medin Medin JH. ${ }^{2}$, Lopez Berruezo J. ${ }^{3}$
}

Sanid. mil. 2011; 67 (2): 100-104; ISSN: 1887-8571

\begin{abstract}
RESUMEN
Presentamos un caso de miopización bilateral aguda asociada a hipertensión ocular por cierre angular, secundario al inicio del tratamiento con topiramato vía oral. Revisión: Revisamos los principales efectos oculares secundarios al tratamiento con topiramato, tales como cierre angular agudo asociado a glaucoma o hipertensión ocular, miopización secundaria, o toxicidad retiniana. Los síntomas aparecen típicamente en las primeras semanas tras iniciar el tratamiento, o al duplicar la dosis. El cuadro se resuelve normalmente al suspender el fármaco. Conclusión: El topiramato es un fármaco utilizado principalmente como antiepiléptico si bien es ampliamente utilizado en otros tratamientos neurológicos y cuadros variados como cefaleas, alcoholismo, espasmos infantiles, neuralgias e incluso tratamiento de la obesidad y bulimia, entre otros. Dados los efectos secundarios descritos a nivel ocular, éstos deben ser conocidos por el oftalmólogo, y considerados por el médico prescriptor, informando al paciente y a su familia al inicio del tratamiento.
\end{abstract}

PALABRAS CLAVE: Topiramato, glaucoma secundario de ángulo cerrado, miopía sencundaria, toxicidad retiniana.

\section{Ocular side effects with topiramate: A case discussion and review of the literature}

SUMMARY

Clinical case: We describe a case of acute bilateral ocular myopia associate to ocular hypertension by angle-closure secondary oral treatment with topiramate. Review: We review the main ocular effects related to the treatment with topiramate, such as angle-closure glaucoma, hypertension, secondary myopia, or retinal toxicity. Symptoms typically occur in the first weeks of the topiramate therapy, or when the dose is duplicate. The disease and symtoms dissapear when the medication is discontinued. Conclusion: The topiramato is a drug used mainly as anti-epileptic, and also in other variable neurological disorders as migraines, alcoholism, spasms, neuralgias and obesity and bulimia, among others. The secondary ocular effects must be known by the ophtalmologist and communicated to the patient and his family at the beginning of the treatment.

KEY WORDS: Topiramate, angle closure glaucoma, secondary miopía, retinal toxicity.

\section{INTRODUCCIÓN}

El Topiramato [sulfamato de 2,3:4,5-Bis-O-(1-metiletilideno)beta-D-fructopiranosa] es un monosacárido sulfamato sustituido sintetizado en 1980 y aprobado en 1995 como medicamento anticonvulsivo utilizado para tratamiento de epilepsia tanto en niños como adultos. Entre otras indicaciones se encuentran principalmente el tratamiento del Síndrome de Lenox-Gastaut, prevención de la migraña, trastorno bipolar $^{1}$, tratamiento de la obesidad (al reducir la ingesta incontrolada) y bulimia nerviosa ${ }^{2}$, alcoholismo ${ }^{3}$, trastornos por estrés postraumático ${ }^{4}$, espasmo infantil ${ }^{5}$, cefalea en racimos $^{6}$, tabaquismo ${ }^{7}$, tratamiento del dolor neuropático, especialmente neuralgia del trigémino ${ }^{8}$ y síndrome piernas inquietas ${ }^{9}$.

Tras su administración oral es rápidamente absorbido, y la mayor parte del fármaco $(70 \%)$ se excreta en la orina sin modificaciones. El resto es metabolizado en hígado por hidroxilación, hidrólisis y glucoronidación. El topiramato estimula los canales de cloro activados por GABA, inhibe los neurotransmisones excitadores a través de acciones sobre el kainato y los receptores AMPA, tiene un efecto

${ }^{1}$ Tcol. Médico. Hospital Militar O’Donnell. Servicio Oftalmología. Ceuta. España.

${ }^{2}$ Lic. Medicina. Facultad Medicina. Santiago de Compostela. España.

${ }^{3}$ DUE. Hospital Militar O’Donnell. Servicio Oftalmología. Ceuta. España.

Dirección para correspondencia: Dr. Juan Medín Catoira. Hospital Militar O'Donnell. Avda. Dr. Marañón s/n. Ceuta. España. Tfno: 956526663. email: jumedcat@telefonica.net

Recibido: 9 de agosto de 2010

Aceptado: 2 de diciembre de 2010 específico sobre los receptores GluR5 del kainato, y actúa como inhibidor de la anhidrasa carbónica.

Los efectos secundarios más frecuentes, presentes en al menos un $10 \%$ de los sujetos, por orden de prevalencia incluyen: dolor de cabeza $(23,8 \%)$, parestesias, aturdimiento y hormigueo $(23,1 \%)$, infecciones del tracto respiratorio superior $(17,5 \%)$, diarrea $(16,8 \%)$, naúseas $(15,4 \%)$, somnonoliencia $(15,4 \%)$, anorexia, pérdida de apetito $(13,3 \%)$, insomnio $(11,9 \%)$, problemas de memoria $(11,2 \%)$, y vértigo $(10,5 \%)$.

\section{CASO CLÍNICO}

Mujer, de 18 años de edad, remitida de urgencias por el servicio de Medicina Interna por referir visión borrosa en ambos ojos de 5 días de evolución. Como únicos antecedentes refiere hipotiroidismo a tratamiento con levotiroxina 100 microgramos/día, y cefaleas para las que se ha prescrito recientemente tratamiento con nadolol $40 \mathrm{mgrs} /$ noches, y topiramato $25 \mathrm{mgrs}$ en las mañanas. Aporta un estudio analítico reciente, previo al tratamiento, dentro de la normalidad.

Su historial clínico oftalmológico recogía una agudeza visual previa, 6 meses antes, de unidad (10/10) ambos ojos con una corrección de $+0,50$ esfera $-0,75$ cilindro a $100^{\circ}$ en ojo derecho y $+0,75$ esfera $-0,75$ cilindro a $75^{\circ}$ en ojo izquierdo.

Se realizó nuevo estudio refractivo que determina un cambio miópico en ambos ojos con $-4,25$ esfera $-0,50$ cilindro a $110^{\circ}$ en 


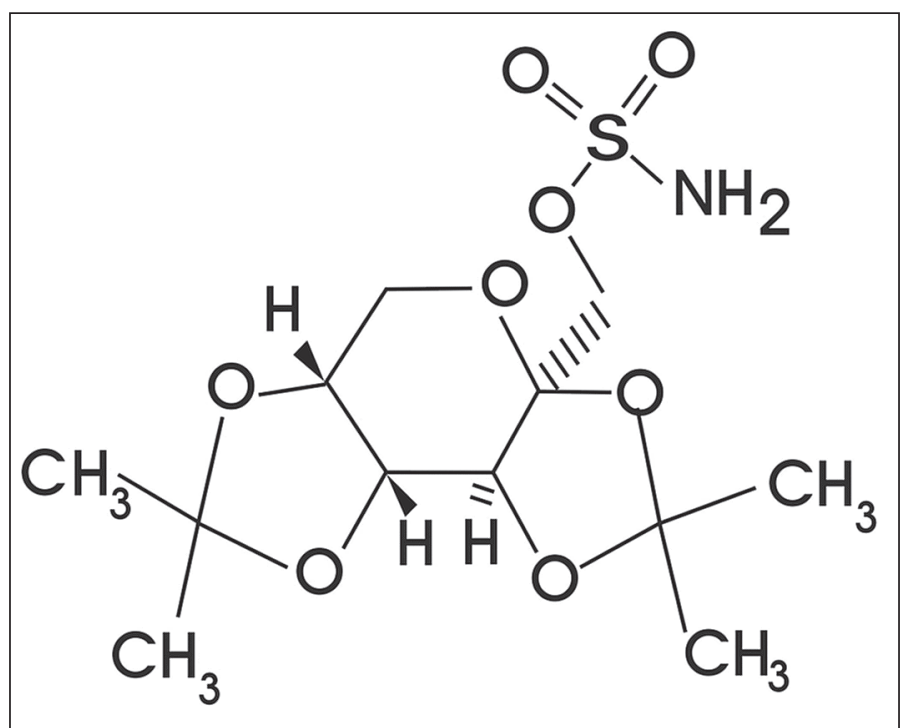

Figura 1. Estructura del Topiramato

ojo derecho y $-3,75$ esfera $-1,00$ cilindro a $60^{\circ}$ en ojo izquierdo, corrección con la que alcanzaba agudeza visual igual a $0,6(6 / 10)$ en ojo derecho y 0,7 (7/10) en ojo izquierdo.

El estudio biomicroscópico mostraba leve hiperemia y congestión conjuntival en ambos ojos, cámaras anteriores estrechas con signo de eclipse positivo (Fig. 2) y córneas y cristalinos transparentes, sin signos inflamatorios ni seclusión. Pupilas isocóricas y normorreactivas. Motilidad ocular normal. La presión intraocular medida con tonómetro de aplanación era de $32 \mathrm{mmHg}$ en el ojo derecho y $29 \mathrm{mmHg}$ en el ojo izquierdo. Exploración de papilas y polo posterior dentro de la normalidad.

Bajo la sospecha de cierre angular agudo se inició tratamiento oral con inhibidores de la anhidrasa carbónica (acetazolamida
$250 \mathrm{mgrs}$ ), $1 / 2 \mathrm{cp}$ cada 8 horas, y tópico con bimatoprost y maleato de timolol en colirio, con suspensión de las medicaciones de reciente introducción (nadolol y topiramato).

A las 24 horas mostraba tensiones de $23 \mathrm{mmHg}$ en ambos ojos, y $13 \mathrm{mmHg}$ en ojo derecho y $12 \mathrm{mmHg}$ en ojo izquierdo a las 48 horas, con normalización de profundidad de la cámara anterior (Fig. 3), por lo que se redujo el tratamiento con acetazolamida progresivamente a las 48 horas, tercer y cuarto día hasta supresión total y el tratamiento tópico se retiró a los 7 días, manteniendo en controles posteriores tensiones normales de $17-18 \mathrm{mmHg}$ en ambos ojos a las 3, 6 y 12 semanas, sin tratamiento.

La visión se normalizó totalmente a las 72 horas, con agudeza visual igual a la unidad en ambos ojos y refracción similar a la previa al episodio.

Se realizó diagnóstico final de miopización e hipertensión ocular por cierre angular agudo secundarios al tratamiento con topiramato, lo cual fue comunicado a la paciente, familia y servicio de Medicina Interna como prescriptor del fármaco.

\section{DISCUSIÓN}

Los efectos secundarios oculares que han sido relacionados con el tratamiento con topiramato, han sido considerados entre las reacciones adversas «raras» incluyen principalmente (fig. 4):

- Cierre Angular.

- Glaucoma Secundario A Cierre Angular.

- Miopía secundaria.

- Alteraciones del Campo Visual

- Maculopatía.

Si bien las reacciones adversas de visión doble y visión anómala, se consideran como muy frecuentes $[\geq 1 / 10]$ en la ficha técnica del producto, las restantes alteraciones oculares son referenciadas como de «rara» presentación $[\geq 1 / 10.000<1 / 1.000]$.
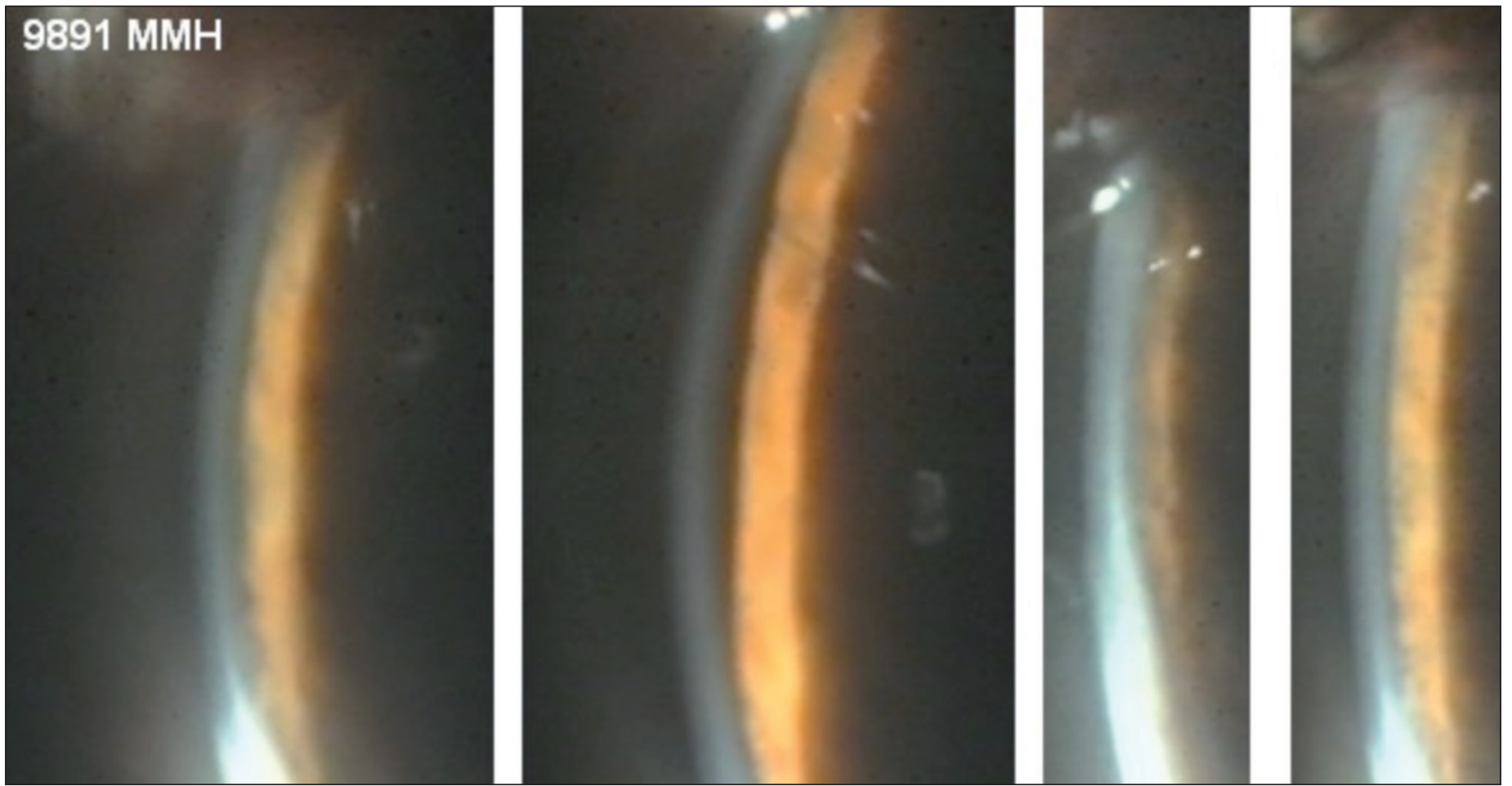

Figura 2. Estrechamiento cámara anterior OD/OI (Medín H.). 


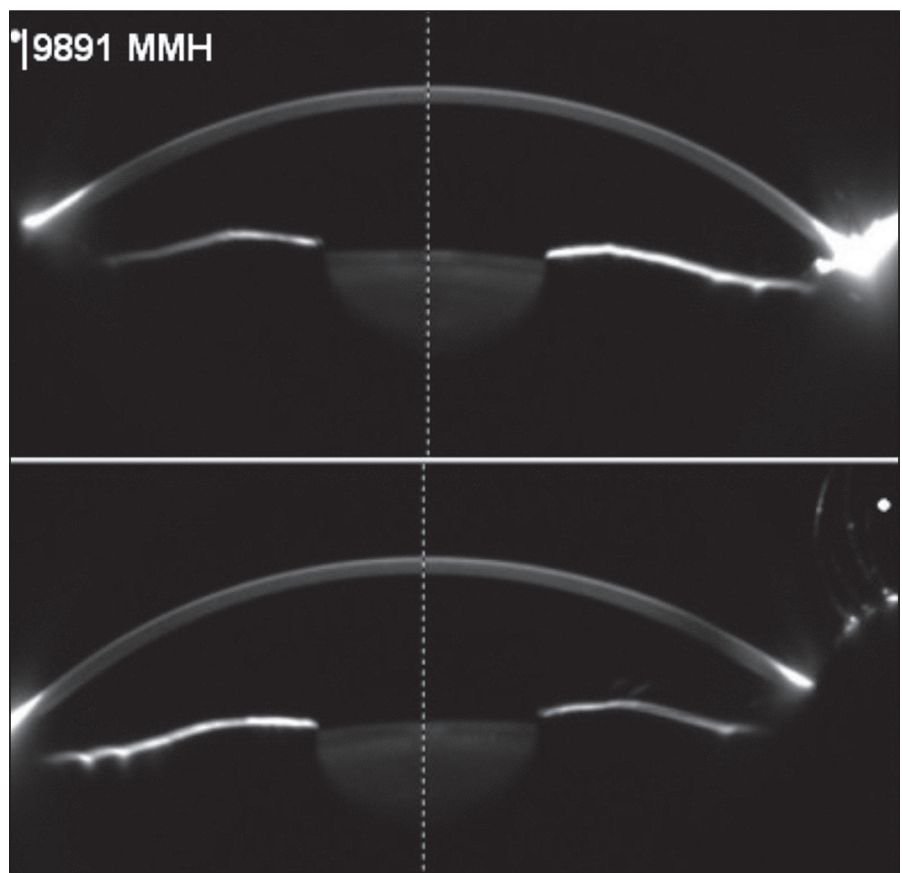

Figura 3. Cámara anterior normal imagen Pentacam (Medín H.).

\section{A. Cierre angular}

Banta y col publicaron en 2001 el primer caso de glaucoma por cierre angular agudo inducido por topiramato en un hombre de 51 años, durante las etapas iniciales de tratamiento ${ }^{10}$. Casos similares fueron posteriormente publicados, entre otros, por Cabrera ${ }^{11} \mathrm{y}$ Kakarla $^{12}$. La mayoría de los cuadros ha tenido lugar dentro de las 2 primeras semanas del inicio del tratamiento o a las pocas horas de duplicar la dosis ${ }^{10,13}$. Las edades de los casos descritos oscilan entre los 3 y 70 años, preferentemente en mujeres ( $80 \%$ de los casos), y errores refractivos previos entre $+4 \mathrm{a}-5,25 \mathrm{D}$. El efecto no parece ser dosis dependiente, pues se han encontrado cambios en tratamiento con dosis bajas de $25 \mathrm{mgrs} / \mathrm{día}^{14}$, como en el caso que nos ocupa.

El cierre angular inducido por topiramato se ha descrito como una reacción idiosincrásica que puede ocurrir incluso en pacientes con cámara anterior de profundidad normal. El fármaco da lugar a un aumento de los niveles de prostaglandinas que actúan desencadenando un edema de cuerpo ciliar a nivel local pero sin actuar a nivel sistémico ${ }^{15}$ aunque se ha comprobado que atraviesa la barrera hematoencefálica y se ha detectado en vítreo ${ }^{16}$. La biomicroscopia ultrasónica muestra la presencia de un síndrome de efusión ciliocoroidea, edema y rotación anterolateral del cuerpo ciliar, con relajación zonular, cambios que conducen a un desplazamiento anterior del diafragma iris-cristalino y, como consecuencia se produce un estrechamiento de la cámara anterior responsable de la miopización y de la crisis de glaucoma agudo ${ }^{17-19}$. Si bien se ha descrito un incremento del grosor del cristalino, este incremento del grosor de la lente contribuye sólo mínimamente (9-16\%) al estrechamiento de la cámara anterior ${ }^{20}$. El efecto no parece ser dosis dependiente pues, como se ha referido, puede darse con dosis de $25 \mathrm{mgrs} /$ día $^{14,21}$.

Otros sulfaderivados que pueden precipitar cierre angular agudo por el mecanismo descrito son acetazolamida, clotrimoxazol, hidroclorotiazida, clortalidona, triamtirene y sulfametoxazol, lo que refuerza la idea de que puede tratarse de una hipersensibilidad al componente sulfamídico 22 .

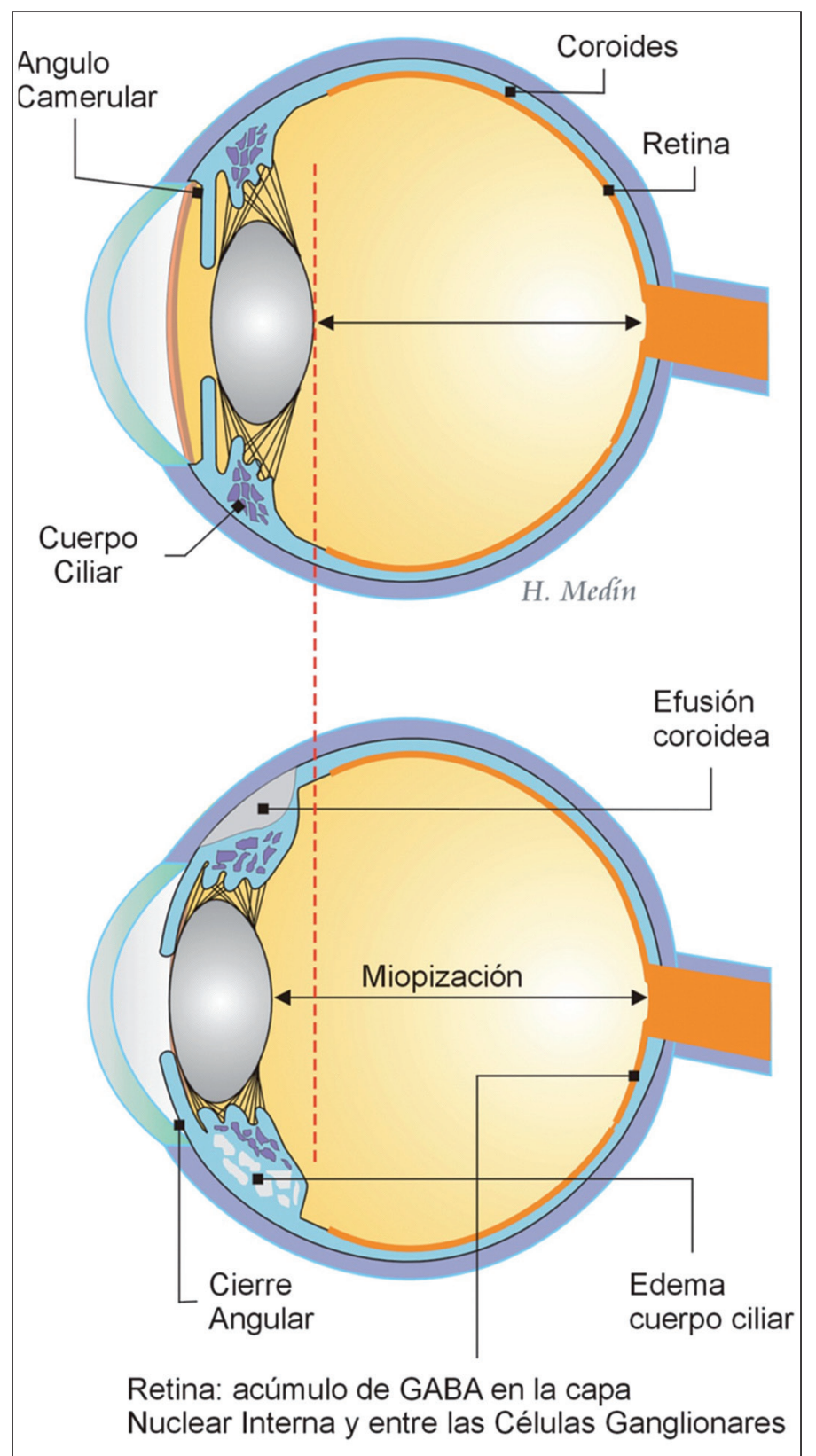

Figura 4. Principales efectos secundarios del topiramato a nivel ocular (Medin H.)

\section{B. Miopia secundaria}

Se han publicado igualmente diferentes casos de miopía secundaria, tanto en adultos como en niños, en un rango de -2 a -9 dioptrías, también con más frecuencia en mujeres ${ }^{23}$, y dentro de los primeros días de tratamiento ${ }^{11,24}$. La presión intraocular puede ser normal, o acompañarse de hipertensión por cierre angular, y el cuadro es reversible dentro de los 3 a 4 días tras retirar el tratamiento ${ }^{25}$.

El mecanismo de la miopía aguda es similar al reportado para las sulfonamidas, fue descrito por vez primera por Berns en $1938^{26} \mathrm{y}$ ha sido comunicado en diferentes casos de tratamiento con topiramato, sulfonamidas y acetazolamida ${ }^{27-31}$. El edema y rotación anterolateral del cuerpo ciliar conducen a la relajación zonular, desplazamiento anterior de iris y cristalino e incremento de la distancia entre lente y retina responsables de la miopización. Si bien se ha descrito también 
Tabla 1. Efectos secundarios relacionados con el topiramato (Medín H.).

\begin{tabular}{|llll|}
\hline Digestivos & Neurológicos & Visuales & Otros \\
\hline Naúseas & Cefaleas & Dolor Periocular & Rash cutáneo \\
Diarrea & Somnoliencia & Visión Borrosa & Cálculos renales \\
Vómitos & Insomnio. & Perdida de Visión Central & Infecciones Tracto Respiratorio Superior \\
Anorexia & Problemas de memoria & Glaucoma & Faringitis. Sinusitis \\
Pérdida de peso & Trastornos de evocación de las & Miopización & Fatiga \\
Cambios del sentido del gusto de & palabras & Cierre Angular & Descenso sudoración \\
\multicolumn{1}{c|}{ bebidas carbonatadas, refrescos } & Falta de concentración & & Hipertermia (especialmente en niños) \\
dietéticos y cerveza & Embotamiento & Desajustes Menstruales \\
Dolor abdominal & Letargia & Dismenorrea \\
Humedad Bucal & Trastornos del habla & Depresión \\
& Desajuste funciones motoras finas & & Aprensibilidad \\
& Pérdida de coordinación & Agitación \\
& Parestesias, hormigueos peribucales y & & Leucopenia \\
& digitales & Osteoporosis \\
& Vértigo & Raquitismo en niños \\
& Convulsiones & Debilidad Muscular \\
& & Dolor de huesos \\
& & Palpitaciones, latidos irregulares \\
\hline
\end{tabular}

la presencia de edema cristaliniano por cambios osmóticos o metabólicos producidos por las sulfonamidas ${ }^{32-34}$, este edema no está presente en todos los casos de miopización por sulfonamidas o análogos.

\section{Alteraciones del campo visual}

Se han descrito casos de hemianopsia homónima tras administración de $125 \mathrm{mg}$ topiramato durante 12 semanas, con mejoría progresiva y recuperación parcial del campo visual al suspender el tratamiento $^{35,36}$. Igualmente se han notificado otros defectos del Campo Visual tales como contracción periférica o defectos arcuatos superiores ${ }^{37}$.

El mecanismo exacto de las alteraciones del campo visual es desconocido. Un estudio en animales evaluando los efectos de la administración crónica de topiramato ha revelado una reducción significativa de la función retiniana con reducción de la amplitud de las ondas b en el ERG asociada a cambios inmunohistquímicos caracterizados por una severa acumulación de GABA en las células amacrinas de la retina interna, plexiforme interna, nuclear interna y entre las células ganglionares. Estos cambios sugieren que el topiramato puede dañar la retina por acumulación de GABA, al igual que ocurre con la vigabatrina ${ }^{38}$.

En 2008, Tsui y colaboradores publican un caso con ERGs anormales electronegativos en ambos ojos, y disminución de las respuestas de los bastones tras tratamiento con topiramato. La presencia de onda $\mathrm{b}$ anormal con onda a normal se ha relacionado clásicamente con la disfunción de los segmentos internos de los fotorreceptores, lo cual parece confirmar los hallazgos de Kjellstrom ${ }^{39}$. ERGs negativos también han sido asociados en otras toxicidades retinianas adquiridas tales como exposición a metanol, hierro, vincristina y quinina ${ }^{40}$.

\section{Otras alteraciones}

Se han descrito otras alteraciones retinianas tales como maculopatía bilateral de carácter irreversible ${ }^{41-43}$, estrías retinianas y desprendimiento de retina ${ }^{44}$. Si bien el mecanismo de la maculopatía es desconocido, se supone sea posiblemente análogo al de la vigabatrina, de probada toxicidad retiniana, al incrementar la presencia y persistencia de altos niveles de GABA en la retina, cuerpo geni- culado lateral y córtex visual. Las estrías retinianas pueden ser causadas por tracción vitreorretiniana media y reversibles tras el cese del tratamiento ${ }^{45}$, y el desprendimiendo de retina podrá ser debido a la presencia de fluido subretiniano por un mecanismo similar a la efusión ciliocoroidea idiopática, que podría precipitar la producción de desgarros sobre zonas degenerativas predisponentes (lattice).

Otras alteraciones que se han asociado al uso del topiramato se encuentran las efusiones supracoroideas y escleritis ${ }^{13}$, edema periorbitario, blefaroespasmo, crisis oculogiras, dolor ocular, diplopía y nistagmus ${ }^{46}$. Estos últimos generalmente en relación con el uso de altas dosis de medicamento.

\section{Manejo}

Dado que en la mayoría de los casos los efectos secundarios oculares se presentan dentro de las pocas semanas de iniciar la dosis, y el $85 \%$ de los casos dentro de las 2 primeras semanas con una media de 7 días, es importante instruir a los pacientes para que informen a su especialista y al médico prescriptor, de cualquier cambio visual o sensación de visión borrosa.

Si bien la miopización será reversible al reducir o suspender el tratamiento, la presencia de cierre angular agudo e hipertensión ocular puede precisar de la asociación de hipotensores tópicos y orales, ciclopléjicos y esteroides. La sospecha de existencia de toxicidad retiniana obligará igualmente a la retirada del tratamiento.

No obstante, la reducción y/o suspensión de la medicación debe realizarse siempre bajo supervisión del médico que prescribió el tratamiento con topiramato, ya que pautas descendentes superiores a $50 \mathrm{mgs}$ podrían exacerbar la patología de base del paciente.

Si bien están indicados los supresores acuosos tópicos y orales, debe tomarse precaución con el uso de la acetazolamida por el riesgo de inducción de cálculos renales y edema ciliar

por lo que se ha propuesto el uso de metilprednisolona y manitol para la rápida resolución del ataque de cierre angular provocado por la efusión cilio-coroidea, al igual que lo descrito en otros glaucomas producidos por efusión uveal ${ }^{47}$.

Se pueden utilizar agentes ciclopléjicos que pueden reducir la presión intraocular mediante la retracción de los procesos ciliares. 
Se desaconseja totalmente el uso de la pilocarpina, que puede incrementar el cierre angular y empeorar los síntomas y signos. La iridotomía, habitualmente útil en el glaucoma por cierre angular puede no ser útil si no existe bloqueo pupilar.

\section{CONCLUSIONES}

Es necesario Informar a los pacientes de los posibles efectos secundarios, antes del inicio del tratamiento con topiramato.

El paciente debe prestar atención a la presencia de potenciales alteraciones visuales al inicio del tratamiento o al incrementar la dosis.

En el estudio de las alteraciones visuales de corta evolución, cierre angular agudo, miopización, maculopatía y alteraciones campimétricas de reciente aparición, el especialista debe recabar información sobre los tratamientos realizados por los pacientes, así como sus posibles reacciones adversas.

En caso de cierre angular agudo, se debe considerar igualmente la presencia de tratamientos asociados con otros sulfaderivados ya $\operatorname{citados}^{22}$.

\section{BIBLIOGRAFÍA}

1. Kusumakar, V.;Lakshmi, N.;Yatham, MB.;O’Donovan, CA.; Kutcher, SP. 152nd Annual Meeting of the American Psychiatric Association. Washington, DC; 1999. Topiramate in rapid cycling bipolar women.

2. Van Ameringen M, Mancini C, Pipe B, Campbell M, Oakman J. Topiramate treatment for SSRI-induced weight gain in anxiety disorders. J Clin Psychiatry. 2002;63:981-984.

3. Johnson BA, Ait-Daoud, Bowden CL, DiClemente C, Roache JD, Lawson K, Javors MA, MA JZ. Oral Topiramate in the treatment of alcohol dependence: a randomised controlled trial. Lancet. 2003;361:1677-85.

4. Berlant JL, Van Kammen DP. Open label topiramate as primary or adjunctive therapy in chronic civilian posttraumatic stress disorder: a preliminary report. J Clin Psychiatry. 2002;63:15-20.

5. Glauser TA, Clark PO, Strawsburg R. A pilot study of topiramate in the treatment of infantile spasms. Epilepsia. 1998 Dec;39(12):1324-8.

6. Laínez MJ, Pascual J, Pascual AM, Santonja JM, Ponz A, Salvador A. Topiramate in the prophylactic treatment of cluster headache. Headache. 2003 Jul-Aug;43(7):784-9.

7. Khazaal Y, Cornuz J, Bilancioni R, Zullino DF. Topiramate for smoking cessation. Psychiatry Clin Neurosci. 2006 Jun;60(3):384-8.

8. Chong MS, Libretto SE. The rationale and use of topiramate for treating neuropathic pain. Clin J Pain. 2003 Jan-Feb;19(1):59-68.

9. Pérez Bravo A. Utilidad del topiramato en el tratamiento del síndrome de piernas inquietas. Actas Esp Psiquiatr 2004; 32(3):132-137

10. Banta JT, Hoffman K, Budenz DL, Ceballos E, Greenfield DS: Presumed topiramateInduced bilateral acute angle-closure Glaucoma. Am J Ophthalmol 2001, 132: 112-4.

11. Cabrera Marrero B, Rodríguez Pérez M, Jerez Olivera E, Crespo Llordens A Rodríguez Melián L, Cabrera López F, Cardona Guerra P. Glaucoma de ángulo cerrado bilateral secundario a topiramato. Arch. Soc. Canar Oftal n 18.

12. Acute bilateral simultaneus angle closure after topiramate administration. Case report. Kakarla V Chalam, Tina Tills, Farhana Syed, Swati Agarwal and Vikram S. Brar. Acute bilateral simultaneus angle closure after topiramate administration. Journal of Medical Case Reports 2008, 2:1

13. Fraunfelder FW, Fraunfelder FT, Reates EU: Topiramate-associated acute, bilateral, secondary angle-closure glaucoma. Ophthalmology 2004, 111:109-11.

14. Sachi D, Vijaya L. Topiramate-induced secondary angle closure glaucoma. J Postgrad Med 2006; 52:72-73

15. Krieg PH, Schipper I. Drug induced ciliary body oedema: a new theory. Eye. 1996:10, 121-126.

16. Moyazani A, Carter J, Nix R. Distribution of topiramate in a medical examiners case. J Anal Toxicol 1999; 23:556-558.

17. Medeiros FA, Zhang XY, Bernd AS, Weinreb RN. Angle closure glaucoma associated with ciliary body detachment in patients using topiramate. Arch. Ophthalmol 2003; 121:282-285
18. Sankar PS, Pasquale LR, Grosskreutz CL. Uveal effusión secondary angle-closure associated with topiramate use. Arch Ophthalmol 2001; 119:1210-11

19. Rhee DJ, Goldberg MJ, Parrish RK Bilateral angle-closure and ciliary body swelling from topiramate. Arch Ophthalmol 2001; 119:1721-3

20. Craig JE, Ong TJ, Louis DL, Wells JM. Mechanism of topiramate-induced acuteonset miopía and angle closure glaucoma. Am J Ohthalmol 2004; 137:193-5.

21. Sachi D, Vijaya L. Topiramate induced secondary angle closure glaucoma. J Postgrad Med 2006; 52:72-73.

22. Tripathi RC, Tripathi BJ, Haggerty D. Drug induced glaucoma. Mechanism and management. Drug Safety 2003; 26:749-67.

23. Bellido Rubiales FJ, Bellido Rubiales JA. Miopía aguda inducida por topiramato. Arch Soc Canar Octal 2005 n $^{\circ} 16$.

24. Bhattacharyya KB, Basu S. Acute miopía induced by topiramate: Report of a case and review of the literature. Neurol India 2005;53:108-9.

25. Vilar Ventura RM, Vilar Fabra C, Ramos Martí F. Miopía Secundaria a tratamiento con topiramato. Neurología 2009; 24 (5):350.

26. Berns W. Proceedings of the meeting of the Swedish Ophthalmological Society, 3rd december 1938. Acta Ophthalmol 1940; 18:96-98 y ha sido descrito en diferentes casos de tratamiento con topiramato, sulfonamidas y acetazolamida.

27. Schear NJ, Rowan AJ, Wiener JA, Haug SJ, Midel JS. Drug-induced myopia: a transient side effect of topiramate. Epilepsia. 1990; 31:643.

28. Bovino JA, Marcus DF. The mechanism of transient sulfonamide therapy. Am J Ophthalmol 1982; 94(1): 99-102.

29. Hook SR, Holaday JT, Prager TC, Goosey JD. Transient myopia induced by sulfonamides. Am J Opthalmol 1986; 101(4):495-6.

30. Garland MA, Sholk A, Guenter KE. Acetazolamide-induced miopía. Am J Obstet Gynecol 1962; 84:69-71.

31. Fan JT, Jonhson DH, Buro RR. Transient miopía, angle-closure glaucoma and choroidal detechmente alter oral acetazolamide. Am J Opthalmol 1993; 115(6).:813-15.

32. Alvaro ME. Effects other than anti-infectious of sulfonamide compounds on eye Arch Ophthalmol 1943; 29:615.

33. Díaz Llopis M, Benítez del Castillo Sánchez JM, Belda JL, Morillas PJ. Toxicidad ocular por fármacos y drogas. IN: Sánchez Salorio M, Díaz LLopis M, Benítez del Castillo JM, Rodríguez Ares MT. Manifestaciones oftalmológcas de las enfermedades generales. Madrid: Sociedad Española de Oftalmología. 2001; 516.

34. Guillamat L. Accidents ophthalmologiques d'origine therapeutique: Encyclopedie Medico Chirurgicale D'ophthalmologie. Paris. Editions Tecniques. 1980; VI 21810 A-101.

35. Asensio-Sánchez VM, Torreblanca-Agüera B, Martínez-Calvo S, Calvo MJ, Rodríguez R. Toxicidad Oftalmológica Severa por Topamax. Arch Soc. Esp Oftalmol 2006; 81:345-348.

36. Foroozan R, Buono LM Foggy visual field defect. Surv Ophthalmol 2003; 48:447-451

37. Mandal A, Chatterjee S, Bose S, Ganguly G. Ocular adverse effects of topiramate: two case reports. Indian J Pharmacol 2008; 40:278-80.

38. Kjellstrom S, Bruun A, Isaksson B, Eriksson T, Andreasson S, Ponjavic V. Retinal function and histopathology in rabits treated with topiramate. Doc Ophthalmol 2006 113:179-86.

39. Tsui I, Casper D, Chou CL, Tsang SH. Electronegative electroretinogram associated with topiramate toxicity and vitelliform maculopathy. Doc Ophthalmol 2008; 116:57-60).

40. HeckenlivelyJR, Arden GB, 2006 principles and practice of clinical electrophysiology of vision. 2nd ed. Cambridge Mass, MIT press 809-822.

41. Asensio-Sánchez VM, Torreblanca-Agüera B, Martínez-Calvo S, Calvo MJ, Rodríguez R. Toxicidad Oftalmológica Severa por Topamax. Arch Soc. Esp Oftalmol 2006; 81:345-348.

42. Vaphiades MS, Mason J. Foggy visual field defect. Surv. Ophthalmol 2004; 49:266-267.

43. Beyenburg S, Weyland C, Reuber M. Presumed topiramate-induced maculopathy Epilepsy \& Behavior. 2009; 14(3): 556-559.

44. Dey KK, Das S, Mishra A, Guha G, Retinal detachment presumably associated with topiramate. Neurol India. 2008; 56:204-5.

45. LIN RC, Cantrill HL, Mieler WF. Retinal toxicities caused by systemic medications. Retinal physicians. http://www.retinalphysician.com/article.aspx?article=101094.

46. Watss P. A review of the ocular side effects of topiramate. 2006. Paediatric Ophthalmology Suncommi-ttee. Royal College of Ophthalmologists. www.rcophth.ac.uk/core/core_picker/download.asp?id=364.

47. Rhee DJ, Ramos-Esteban JC, Nipper KS. Rapid resolution of topiramate induced angle-closure with methylprednisolone and mannitol. Am J Opthalmol 2006; 141(6):1133-4). 